\title{
Practical preparation procedures for docetaxel-loaded nanoparticles using polylactic acid-co-glycolic acid
}

This article was published in the following Dove Press journal:

International Journal of Nanomedicine

6 October 2011

Number of times this article has been viewed

\author{
Chang-Gu Keum ${ }^{*}$ \\ Young-Wook Noh ${ }^{*}$ \\ Jong-Suep Baek' \\ Ji-Ho Lim' \\ Chan-Ju Hwang' \\ Young-Guk $\mathrm{Na}^{\prime}$ \\ Sang-Chul Shin ${ }^{2}$ \\ Cheong-Weon Cho'
}

'College of Pharmacy and Institute of Drug Research and Development,

Chungnam National University,

Gungdong, Yuseonggu, Daejeon,

South Korea; ${ }^{2}$ College of Pharmacy,

Chonnam National University,

Yongbongdong, Buggu, Gwangju,

South Korea

*These authors contributed equally to this work
Correspondence: Cheong-Weon Cho College of Pharmacy and Institute of Drug Research and Development,

Chungnam National University,

220 Gungdong, Yuseonggu,

Daejeon 305-764, South Korea

Tel +824282I 5934

Fax +82428236566

Email chocw@cnu.ac.kr
Background: Nanoparticles fabricated from the biodegradable and biocompatible polymer, polylactic-co-glycolic acid (PLGA), are the most intensively investigated polymers for drug delivery systems. The objective of this study was to explore fully the development of a PLGA nanoparticle drug delivery system for alternative preparation of a commercial formulation. In our nanoparticle fabrication, our purpose was to compare various preparation parameters.

Methods: Docetaxel-loaded PLGA nanoparticles were prepared by a single emulsion technique and solvent evaporation. The nanoparticles were characterized by various techniques, including scanning electron microscopy for surface morphology, dynamic light scattering for size and zeta potential, $\mathrm{x}$-ray photoelectron spectroscopy for surface chemistry, and high-performance liquid chromatography for in vitro drug release kinetics. To obtain a smaller particle, $0.2 \%$ polyvinyl alcohol, $0.03 \% \mathrm{D}$ - $\alpha$-tocopheryl polyethylene glycol 1000 succinate (TPGS), 2\% Poloxamer 188, a five-minute sonication time, $130 \mathrm{~W}$ sonication power, evaporation with magnetic stirring, and centrifugation at $8000 \mathrm{rpm}$ were selected. To increase encapsulation efficiency in the nanoparticles, certain factors were varied, ie, 2-5 minutes of sonication time, 70-130 W sonication power, and 5-25 mg drug loading.

Results: A five-minute sonication time, $130 \mathrm{~W}$ sonication power, and a $10 \mathrm{mg}$ drug loading amount were selected. Under these conditions, the nanoparticles reached over $90 \%$ encapsulation efficiency. Release kinetics showed that $20.83 \%$, $40.07 \%$, and $51.5 \%$ of the docetaxel was released in 28 days from nanoparticles containing Poloxamer 188, TPGS, or polyvinyl alcohol, respectively. TPGS and Poloxamer 188 had slower release kinetics than polyvinyl alcohol. It was predicted that there was residual drug remaining on the surface from $\mathrm{x}$-ray photoelectron spectroscopy.

Conclusion: Our research shows that the choice of surfactant is important for controlled release of docetaxel.

Keywords: docetaxel, nanoparticles, poly (lactic-co-glycolic acid), formulation variables, sonication, encapsulation efficiency

\section{Introduction}

Docetaxel is an anticancer agent of the taxoid family. An analog of paclitaxel, docetaxel was obtained by semisynthesis from 10-deacetyl baccatin III extracted from the needles of the European yew tree, Taxus baccata $L,{ }^{1,2}$ and is more effective than paclitaxel as an inhibitor of microtubule polymerization. Docetaxel combined with certain other chemotherapeutic drugs shows high anticancer efficacy in breast, pancreatic, gastric, and urothelial carcinoma. Docetaxel has very low water solubility, and presently the only available formulation for clinical use consists of a solution 
$(40 \mathrm{mg} / \mathrm{mL})$ in a vehicle containing a high concentration of polysorbate 80 . This vehicle has been associated with several hypersensitivity reactions and exhibits incompatibility with common polyvinyl chloride intravenous administration sets. ${ }^{3}$ In order to eliminate the polysorbate 80 -based vehicle and to increase drug solubility, alternative dosage forms have been suggested, including liposomes ${ }^{4,5}$ and cyclodextrins. ${ }^{6}$

The polymer matrix of nanoparticles must meet several requirements, such as biocompatibility, biodegradability, mechanical strength, and ease of processing. The best known class of biodegradable materials for controlled release is poly (lactic acid-co-glycolic acid) (PLGA). ${ }^{7}$ Many publications have described preparation of nanoparticles using PLGA and studied formulation variables, including the molecular weight of PLGA, ${ }^{8}$ lyophilizing agents, ${ }^{9}$ and surfactants. ${ }^{10}$ Therefore, we investigated a sustained injection formulation of docetaxel prepared using PLGA by the solvent-evaporation method. ${ }^{11}$

Formation of an emulsion is the most important step in preparation of nanoparticles because the size of the emulsion droplets is directly related to the final nanoparticle size. This emulsion, which is formed by mixing an organic phase consisting of polymer with an aqueous phase containing a surfactant or stabilizer, is broken down into droplets by applying external energy, and the nanodroplets lead to nanoparticle formation upon evaporation of the organic solvent. ${ }^{12}$

In this study, we focused on the details for preparation of docetaxel nanoparticles using PLGA. To obtain a small particle size and narrow size distribution, we changed some important parameters according to emulsification and evaporation. The primary purpose was to identify the optimal preparation conditions for nanoparticles of docetaxel using PLGA.

\section{Materials and methods Materials}

PLGA (50:50 Resomer RG 502H) with free carboxyl end groups was obtained from Boehringer Ingelheim (Ingelheim, Germany). Docetaxel was a gift from SamyangGenex Corporation (Daejeon, Korea). Polyvinyl alcohol purchased from Sigma-Aldrich (Steinheim, Switzerland) was 87\%-89\% hydrolyzed and had a molecular weight of 146,000-186,000. Poloxamer 188 was purchased from BASF (Ludwigshafen, Germany). D- $\alpha$-tocopheryl polyethylene glycol 1000 succinate (TPGS) and sucrose were purchased from SigmaAldrich. All other chemical reagents were commercial products of analytical or reagent grade and were used without further purification.

\section{Nanoparticle preparation}

Nanoparticles containing docetaxel were fabricated by a single emulsion technique and solvent evaporation. Briefly, docetaxel was dissolved in $2 \mathrm{~mL}$ of dichloromethane and then $100 \mathrm{mg}$ of PLGA was added into the solution. This oil phase was emulsified for two minutes with a probe sonicator (VCX 130, Sonic and Materials, Newtown, CT) at $100 \mathrm{~W}$ in an ice bath. The resulting oil solution was further emulsified into $40 \mathrm{~mL}$ of aqueous solution containing $0.2 \%$ polyvinyl alcohol for 10 minutes in an ice bath with sonication. After emulsification, the oil-in-water emulsion was magnetically stirred for eight hours to evaporate the organic solvent. The nanoparticles formed were isolated by centrifugation for 20 minutes at 8000 rpm. Finally, the nanoparticles were washed with distilled water to remove the residual surfactant and then freeze-dried in the presence of sucrose. The effects of the various processing parameters on hydrodynamic particle size and percentage drug encapsulation efficiency were studied. The processing parameters included surfactant concentration and type in the aqueous phase, initial drug loading, and sonication pulse time and power. Unless otherwise mentioned, all experiments were conducted by varying one parameter while keeping all other process parameters constant.

\section{Docetaxel assay}

The freeze-dried nanoparticles containing docetaxel were weighed and dissolved in dichloromethane. After vortexing for 15 minutes, dichloromethane was evaporated with nitrogen gas. Yield, drug loading, and encapsulation efficiency were calculated as follows:

$$
\begin{aligned}
\text { Yield }(\%)= & \text { weight of particles } \\
& / \text { weight of feeding polymer and drug } \\
& \times 100
\end{aligned}
$$

Drug loading $(\%)=$ weight of drug in particles /weight of particles $\times 100$

Encapsulation efficiency $(\%)=$ weight of drug in particles /weight of feeding drugs $\times 100$

\section{Particle size, zeta potential, and surface tension measurement}

Size analysis of the nanoparticles was performed using a dynamic light scattering analyzer (ELS-8000, Otasuka Electronics, Tokyo, Japan). The nanoparticles containing docetaxel was added to the sample dispersion unit and sonicated in order to minimize interparticle interactions. 
The obscuration range was maintained at $2 \%-50 \%$. The instrument was set to measure the sample 30 times, and the average volume diameter was obtained. In order to measure the zeta potential of the nanoparticles, an aqueous dilute suspension of nanoparticles containing docetaxel was prepared and sonicated for one minute. The surface tension of the nanoparticles containing docetaxel was also checked (Sigma 703D, Attension/Biolin Scientific, Espoo, Finland) according to the concentration of surfactants.

\section{Scanning electron microscopy}

Scanning electron microscopy was used to verify uniformity of particle shape and size. The lyophilized nanoparticles were dropped onto double-sided carbon tape. They were then vacuum-coated for 45 seconds with a platinum mixture and examined for morphology with a FE-SEM (JEOL JSM7500, Thermo Scientific) at $5 \mathrm{kV}$.

\section{FTIR spectroscopy and x-ray photoelectron spectroscopy}

Surface structure characteristics of the nanoparticles containing docetaxel were determined in the solid state using Fourier transform infrared spectroscopy (FTIR, Nicolet 380, Thermo Scientific). Nanoparticle samples were scanned in the infrared range of $400-4000 \mathrm{~cm}^{-1}$. The surface chemistry of the nanoparticles containing docetaxel was checked by x-ray photoelectron spectroscopy (Multilab 2000, Thermo Scientific), and the survey spectrum recorded covered a binding energy range from 0 to $1200 \mathrm{eV}$ using a pass energy of $80 \mathrm{eV}$. Curve fitting was performed using the software supplied by the manufacturer.

\section{In vitro release profiles from docetaxel-loaded nanoparticles}

Studies of docetaxel release from the nanoparticles containing docetaxel were conducted at room temperature in triplicate using a semipermeable membrane (molecular weight cutoff 10,000 Da, MEMBRA-CEL, Chicago, IL) to separate the donor and receptor media. The receptor medium was filled with $100 \mathrm{~mL}$ of $\mathrm{pH} 7.4$ phosphate-buffered solution to ensure solubility. A mass of nanoparticles, equivalent to $300 \mu \mathrm{g}$ of docetaxel, was dispersed in the donor medium $(5 \mathrm{~mL})$, and $1 \mathrm{~mL}$ samples were withdrawn from the receiver medium at a defined time point and replaced with an equivalent volume of fresh medium.

\section{Statistical analysis}

The Student's $t$-test was used to compare groups. A $P$ value $<0.05$ was considered to be statistically significant.

\section{Results and discussion}

Factors affecting diameter of docetaxel-loaded PLGA nanoparticles

The effect of variables in the preparation steps on the mean diameter of the docetaxel-loaded PLGA nanoparticles was investigated. These variables included the organic solvents, concentration of polyvinyl alcohol, TPGS, and Poloxamer 188 in the aqueous phase, sonication time and power, evaporation method, and centrifugation speed.

\section{Effect of various organic solvents and surfactants}

The formation of an emulsion is the most important step in nanoparticle preparation because the size of the emulsion droplets is directly related to the final nanoparticle size. This emulsion, which is formed by mixing an organic phase consisting of polymer with an aqueous phase containing a surfactant or stabilizer, is broken down into droplets by applying external energy, and these nanodroplets lead to nanoparticle formation upon evaporation of the organic solvent. ${ }^{12}$

First, we determined the optimal concentration of surfactant, ie, polyvinyl alcohol, TPGS, or Poloxamer 188, in the aqueous phase. The concentration of these surfactants was varied to identify the effect on particle size, keeping the other parameters constant as in the standard procedure. ${ }^{13}$ Figure 1 shows the nanoparticle size and surface tension according to the concentration of each surfactant in the aqueous phase. The surfactant concentration in the aqueous phase was varied from $0.1 \%$ to $4 \%$ for polyvinyl alcohol, $0.015 \%$ to $1 \%$ for TPGS, or $0.1 \%$ to $4 \%$ for Poloxamer 188 ; all other processing parameters, including sonication time and drug concentration, were kept constant. An increase in polyvinyl alcohol concentration caused a significant decrease in nanoparticle size at $0.2 \%$ in accordance with the surface tension. This was consistent with a low interfacial tension that was reduced so that particle size was decreased. ${ }^{14}$ Particle size then increased with concentrations above $0.2 \%$ polyvinyl alcohol. Other researchers have reported that increasing the polyvinyl alcohol concentration increases the viscosity of the external aqueous phase, resulting in a decrease in net shear stress and a corresponding increase in particle size. ${ }^{15}$ A particle size of $44.3 \pm 0.7 \mu \mathrm{m}$ was found with $4 \%$ Poloxamer 188 , and a size of $46.5 \pm 1.2 \mu \mathrm{m}$ was found with $2 \%$ Poloxamer 188 . However, when using TPGS in the nanoparticle preparation, surface tension and particle size did not show any correlation. 

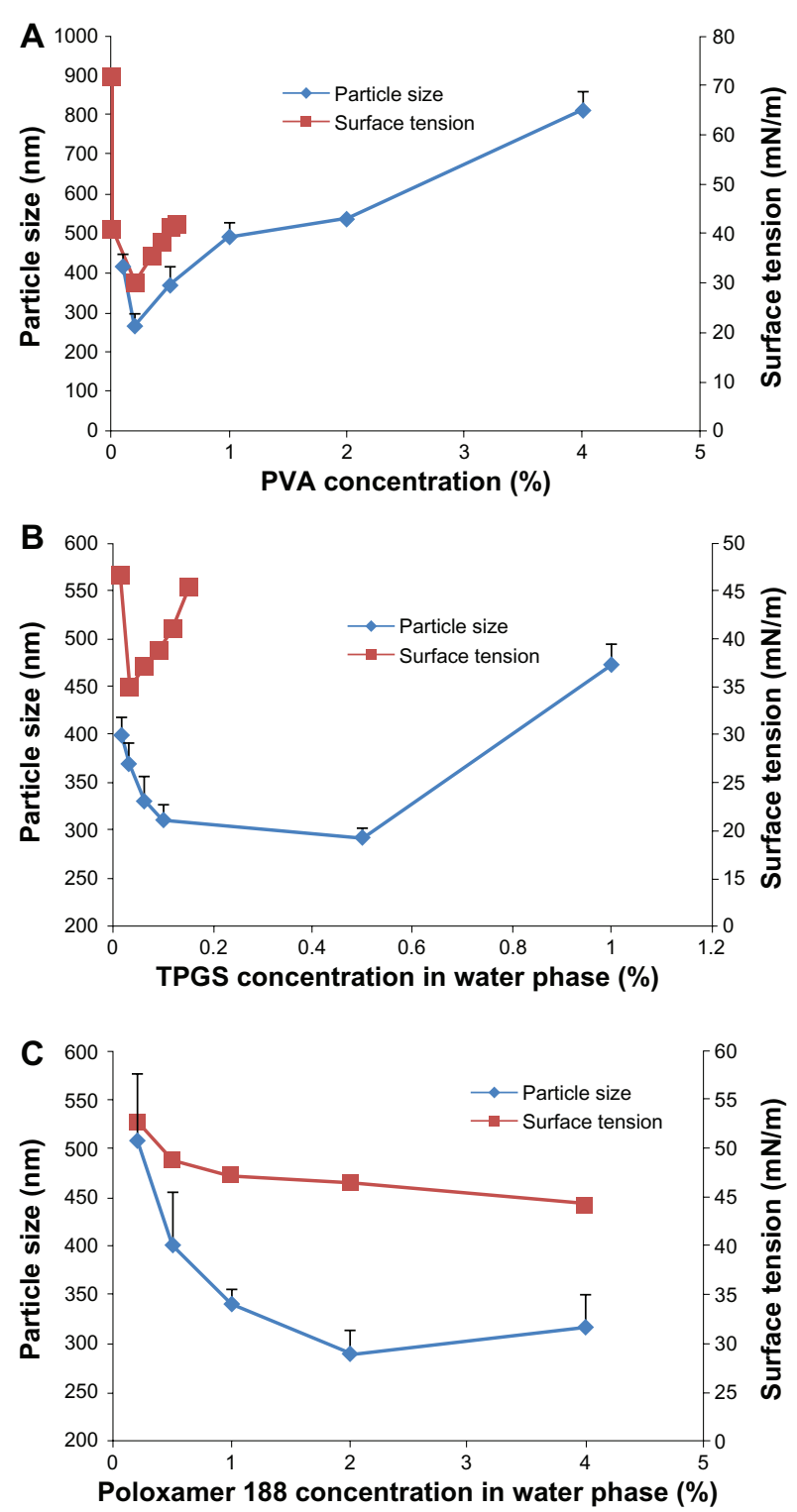

Figure I Particle size and surface tension of each formulation with various organic solvents and surfactants. (A) PVA, (B) TPGS, and (C) Poloxamer 188.

Abbreviations: PVA, polyvinyl alcohol; TPGS, D- $\alpha$-tocopheryl polyethylene glycol 1000 succinate.

The predetermined concentration of surfactants was $2 \%, 0.03 \%$, and $0.2 \%$ for polyvinyl alcohol, TPGS, and Poloxamer 188, respectively, in previous experiments. Subsequently, three kinds of organic solvents, including dichloromethane, chloroform, and ethyl acetate were used. With dichloromethane, the size of the nanoparticles was $290.2 \mathrm{~nm}, 292.8 \mathrm{~nm}$, and $265.5 \mathrm{~nm}$ for Poloxamer 188, TPGS, and polyvinyl alcohol, respectively, and the zeta potential was $-19.27 \mathrm{mV},-3.29 \mathrm{mV}$, and $-16.06 \mathrm{mV}$, respectively. With chloroform, the nanoparticles showed a size of $340.5 \mathrm{~nm}, 531.0 \mathrm{~nm}$, and $274.4 \mathrm{~nm}$ with Poloxamer 188 , TPGS, and polyvinyl alcohol, respectively, and the zeta potential was $18.94 \mathrm{mV},-20.39 \mathrm{mV}$, and $-16.44 \mathrm{mV}$, respectively. With ethyl acetate, the size of the nanoparticles was $438.8 \mathrm{~nm}, 1044.2 \mathrm{~nm}$, and $393.6 \mathrm{~nm}$ with Poloxamer 188 , TPGS, and polyvinyl alcohol, respectively, and the zeta potential was $-20.75 \mathrm{mV},-14.52 \mathrm{mV}$, and $-19.2 \mathrm{mV}$, respectively (Figure 2). Based on these results, dichloromethane as the organic phase and $0.2 \%$ polyvinyl alcohol as the aqueous phase showed the smallest particle size. It has also been reported that the role of a surfactant is to stabilize the emulsion droplets by preventing them from coalescing. For effective stabilization, the surfactant molecules have to cover the interface between the organic solvent and the aqueous phase for all the droplets. ${ }^{12}$

\section{Effect of sonication time and power}

The smallest nanoparticles were obtained with sonication for five minutes at $130 \mathrm{~W}$ (Table 1). Thus, the sonication power was likely to decrease the nanoparticle size, probably because the sonication energy also increased the energy released by emulsification and decreased the mean particle diameter.

\section{Effect of evaporation method and time}

The method used to remove the organic solvent had a significant influence on nanoparticle size. We obtained a submicron particle size with a Rotavapor and magnetic stirring, but it was impossible to obtain a submicron-sized nanoparticle in nitrogen gas and reduced pressure. Table 2 shows that nitrogen gas had the fastest evaporation rate for organic phase removal, but also resulted in a larger particle size than with magnetic stirring. It could be suggested that the evaporation rate for removing organic solvent is not an important parameter. It is countered that increasing evaporation rate of organic solvent influences particle size. ${ }^{16}$ Figure 3 shows the effect of different magnetic stirring times on removal of the organic solvent during preparation of the nanoparticles. With two, four, and six hours of stirring, nanoparticles were aggregated but showed a spherical shape. Therefore, we concluded that a minimum of eight hours of evaporation with magnetic stirring is needed to remove the organic solvent.

\section{Effect of centrifugation speed}

Figure 4 shows nanoparticles prepared at different centrifugation speeds. At 8000 and 10,000 rpm, the nanoparticles had a spherical shape and did not aggregate, but aggregated at 13,000 and 15,000 rpm. Comparing 8000 and $10,000 \mathrm{rpm}$, large nanoparticles existed with small particles at 10,000 rpm. 


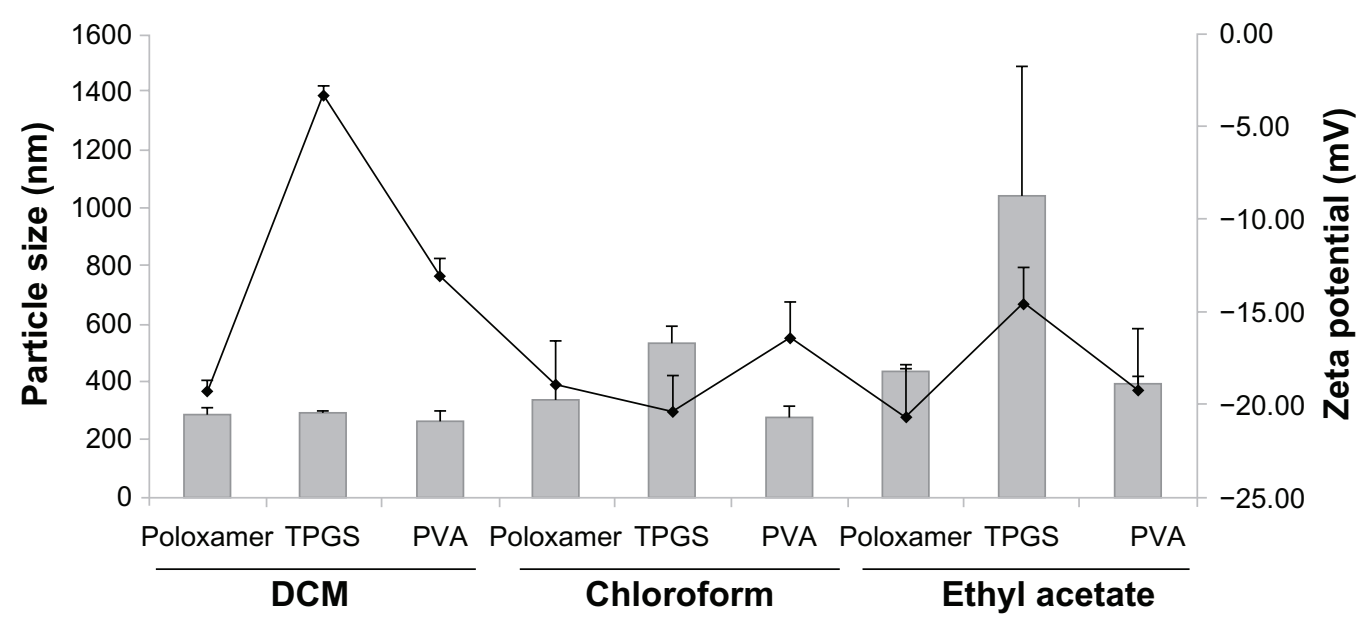

Figure 2 Particle size and zeta potential of each formulation with various organic solvents and surfactants.

Abbreviations: PVA, polyvinyl alcohol; TPGS, D- $\alpha$-tocopheryl polyethylene glycol 1000 succinate; DCM, dichloromethane.

\section{Factors affecting encapsulation efficiency of docetaxel-loaded nanoparticles}

The effect of variables in the preparation steps on the encapsulation efficiency of docetaxel-loaded PLGA nanoparticles was investigated. These included the mixing order of the organic phase, drug amount, sonication time and power, types of organic solvents, and concentration of polyvinyl alcohol, TPGS, or Poloxamer 188 in the aqueous phase.

\section{Effect of mixing order for organic phase}

To optimize the amount of docetaxel-loaded PLGA nanoparticles, various experimental conditions for the preparation of these particles were evaluated. The influence of preparation of the organic phase on the particles was evaluated, and the effect on the encapsulation efficiency of docetaxel is given in Table 3. Only $3.64 \%-10.03 \%$ of the initial drug load was encapsulated in the nanoparticles, except when the organic phase was prepared using F4, which gave a $78.78 \%$ encapsulation efficiency. Therefore, F4 was selected for preparation of the organic phase. This may have resulted from a different interaction between docetaxel and the polymer occurring when they are both solubilized, thus leading to different encapsulation in the nanoparticles. ${ }^{17} \mathrm{~F} 4$ and the other preparations also have different polymer concentrations and mixing methods, and an interaction between drug and polymer may occur.

\section{Effect of drug amount}

Table 4 shows the effect of initial drug amount on encapsulation efficiency. As the initial amount of drug increased, the encapsulated drug first increased and then decreased with an initial drug amount of $25 \mathrm{mg}$. The maximum encapsulated drug amount was $10 \mathrm{mg}$ of docetaxel. In our experiments, as the initial drug amount increased during formulation, the drug concentration in the organic phase also increased. This means that more drug molecules could interact with the PLGA molecules, resulting in an increase in the amount of docetaxel encapsulated. However, the increase in encapsulation amounts was not proportional to the increase in initial drug amounts during formulation, so the encapsulation efficiency decreased.

Table I Effects of sonication time and power on nanoparticle characteristics

\begin{tabular}{|c|c|c|c|}
\hline $\begin{array}{l}\text { Sonication time (minutes) } \\
\text { and power }(\mathrm{W})\end{array}$ & $\begin{array}{l}\text { Particle size, } \\
\text { mean } \pm \text { SD }(\mathrm{nm})\end{array}$ & $\begin{array}{l}\text { Zeta potential, } \\
\text { mean } \pm S D(\mathrm{mV})\end{array}$ & $\begin{array}{l}\text { Encapsulation efficiency, } \\
\text { mean } \pm \text { SD (\%) }\end{array}$ \\
\hline 2,70 & $514.5 \pm 53.1$ & $-4.64 \pm 0.88$ & $87.56 \pm 3.51$ \\
\hline 2,100 & $422.0 \pm 22.1$ & $-9.37 \pm 0.76$ & $57.75 \pm 5.67$ \\
\hline 2,130 & $380.6 \pm 42.4$ & $-8.63 \pm 2.64$ & $75.41 \pm 3.25$ \\
\hline 5,70 & $478.6 \pm 60.2$ & $-4.39 \pm 1.52$ & $74.04 \pm 3.87$ \\
\hline 5,100 & $290.1 \pm 35.6$ & $-12.45 \pm 2.37$ & $77.41 \pm 8.67$ \\
\hline 5,130 & $234.5 \pm 20.1$ & $-11.40 \pm 1.65$ & $89.83 \pm 2.75$ \\
\hline 10,70 & $498.7 \pm 45.4$ & $-2.54 \pm 1.89$ & $60.69 \pm 8.48$ \\
\hline 10,100 & $423.9 \pm 41.6$ & $-7.52 \pm 0.78$ & $79.90 \pm 6.44$ \\
\hline 10,130 & $427.5 \pm 16.4$ & $-7.56 \pm 2.21$ & $93.31 \pm 1.56$ \\
\hline
\end{tabular}

Abbreviation: SD, standard deviation. 
Table 2 Effect of evaporation equipment on size of nanoparticles

\begin{tabular}{lc}
\hline Solvent evaporation method & $\begin{array}{l}\text { Particle size } \\
\text { Mean } \pm \text { SD (nm) }\end{array}$ \\
\hline Rotavapor (I hour) & $776.9 \pm 39.5$ \\
Stirring overnight (8 hours) & $563.6 \pm 24.8$ \\
Nitrogen gas (30 minutes) & $1284.3 \pm 115.7$ \\
Reduced pressure (I hour) & $2569.7 \pm 909.7$ \\
\hline
\end{tabular}

Abbreviation: SD, standard deviation.

\section{Effect of extent of sonication}

Table 1 shows the effects of different sonication parameters on encapsulation efficiency. For five and 10 minutes, encapsulation efficiency increased with increasing sonication power. However, for two minutes, the higher energy released in the process led to low encapsulation efficiency. These results suggest that increased sonication power with five or 10 minutes leads to more encapsulated nanoparticles. With two minutes of sonication, a sonication power above $70 \mathrm{~W}$ was too high to make a stable nanosuspension.

\section{Effect of various organic solvents and surfactants}

Figure 5 shows the encapsulation efficiencies of docetaxel. The encapsulation efficiency of docetaxel decreased significantly with chloroform. The same solvent prepared with TPGS led to the lowest encapsulation efficiency, and polyvinyl alcohol led to the highest encapsulation efficiency.
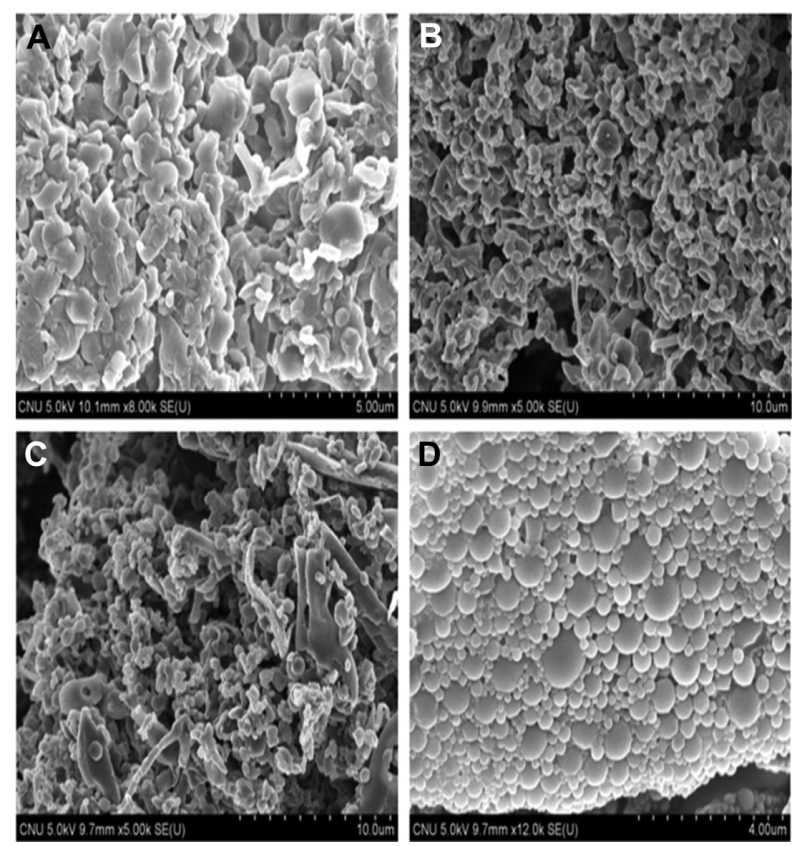

Figure 3 Scanning electron microscopic images of polylactic acid-co-glycolic acid nanoparticles with various magnetic stirring times to remove organic solvent. (A), two hours, (B), four hours, (C), six hours, and (D) eight hours.
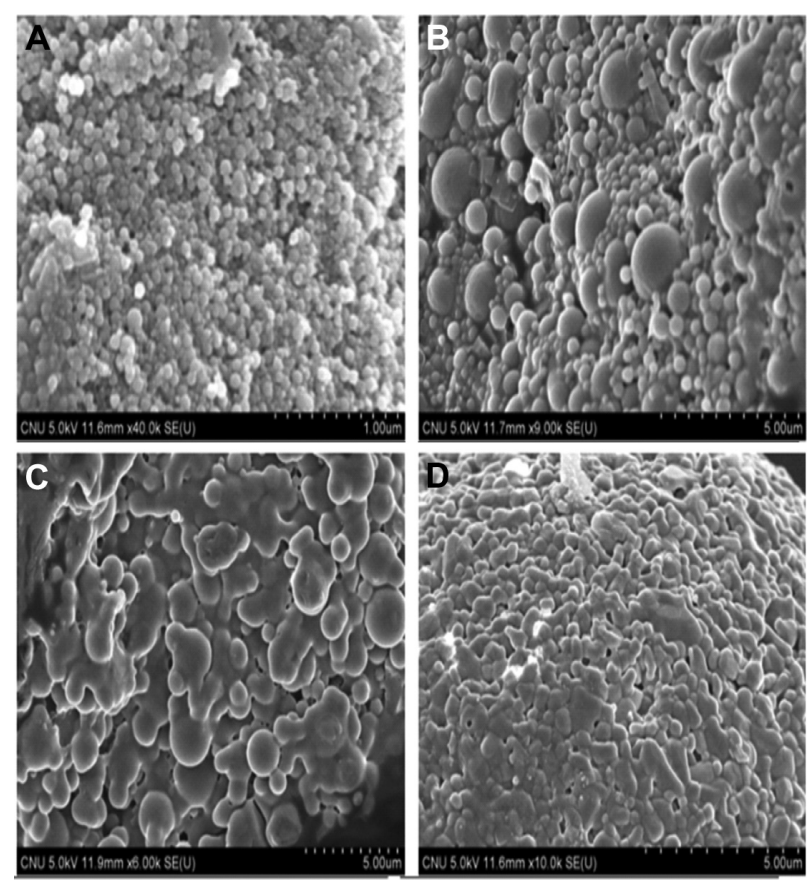

Figure 4 Scanning electron microscopic images of polylactic acid-co-glycolic acid nanoparticles with different centrifugation speeds. (A) 8000, (B) 10,000, (C) 13,000, and (D) 15,000 .

\section{X-ray photoelectron spectroscopy and FTIR analysis}

$\mathrm{X}$-ray photoelectron spectroscopy is a quantitative technique that gives the elemental and averaged chemical composition by measuring the binding energy of electrons associated with atoms over a $5-10 \mathrm{~nm}$ depth inside the polymeric matrix. ${ }^{18}$ $\mathrm{X}$-ray photoelectron spectroscopy $\mathrm{C} 1 \mathrm{~s}$ (atomic orbital $1 \mathrm{~s}$ of carbon) envelopes on the surface of docetaxel-loaded

Table 3 Encapsulation efficiency according to mixing order of docetaxel and PLGA

\begin{tabular}{lll}
\hline Formulation & $\begin{array}{l}\text { Mixing order of docetaxel } \\
\text { and PLGA }\end{array}$ & $\begin{array}{l}\text { Encapsulation } \\
\text { efficiency } \\
\text { Mean } \pm \text { SD (\%) }\end{array}$ \\
\hline FI & $\begin{array}{l}\text { Docetaxel and PLGA were } \\
\text { solubilized in DCM (vortexed) } \\
\text { Focetaxel and PLGA were }\end{array}$ & $10.03 \pm 1.68$ \\
solubilized in DCM (sonicated) & $4.13 \pm 2.31$ \\
F3 & $\begin{array}{l}\text { PLGA was added into docetaxel- } \\
\text { DCM solution and add DCM } \\
\text { (vortexed) }\end{array}$ & \\
F4 & $\begin{array}{l}\text { PLGA was added into docetaxel- } \\
\text { DCM solution and add DCM } \\
\text { (sonicated) }\end{array}$ & $78.78 \pm 6.13$ \\
F5 & $\begin{array}{l}\text { Docetaxel-DCM solution mixed } \\
\text { with PLGA-DCM solution } \\
\text { (sonicated) }\end{array}$ \\
\hline
\end{tabular}

Abbreviations: SD, standard deviation; DCM, dichloromethane; PLGA, polylacticco-glycolic acid. 
Table 4 Effect of drug amount on nanoparticle characteristics

\begin{tabular}{llll}
\hline Drug amount (mg) & $\begin{array}{l}\text { Particle size, } \\
\text { mean } \pm \text { SD }(\mathbf{n m})\end{array}$ & $\begin{array}{l}\text { Zeta potential, } \\
\text { mean } \pm \text { SD }(\mathbf{m V})\end{array}$ & $\begin{array}{l}\text { Encapsulation efficiency, } \\
\text { mean } \pm \text { SD (\%) }\end{array}$ \\
\hline 0 & $232.8 \pm 11.6$ & - & - \\
5 & $286.3 \pm 9.4$ & $-17.7 \pm 1.3$ & $60.5 \pm 1.9$ \\
10 & $269.4 \pm 2.9$ & $-8.4 \pm 1.6$ & $97.4 \pm 4.8$ \\
15 & $282.1 \pm 10.2$ & $-4.9 \pm 1.4$ & $61.1 \pm 13.7$ \\
20 & $282.1 \pm 23.4$ & $5.9 \pm 2.3$ & $49.7 \pm 8.6$ \\
25 & $285.3 \pm 11.7$ & $13.8 \pm 3.1$ & $38.7 \pm 2.9$ \\
\hline
\end{tabular}

Abbreviation: SD, standard deviation.

nanoparticles and its components were examined and the results are displayed in Table 5. Firstly, there was a nitrogen signal detected in the physical mixture and nanoparticles, which could mean that docetaxel was on the surface. The nitrogen signal found only in docetaxel could be evidence of docetaxel existing on the surface of nanoparticles. ${ }^{18}$

The infrared spectra of the docetaxel nanoparticles are shown in Figure 6. The major peak of docetaxel was around $1725 \mathrm{~cm}^{-1}$ (ester stretching) and PLGA showed the characteristic vibration of carboxylic acid at $1750 \mathrm{~cm}^{-1}$. FTIR spectra for the physical mixture showed a major peak of docetaxel and PLGA. However, in the FTIR spectra for docetaxel nanoparticles, the major peak of docetaxel was significantly lowered. This indicated a chemical interaction between docetaxel and PLGA, ${ }^{19}$ by which the carboxylic acid in PLGA interacted with the ester group in docetaxel, and the ester absorption frequency was correspondingly lowered. ${ }^{20}$

\section{In vitro release with various surfactants and organic solvents}

Figure 7A shows the in vitro release kinetics for the three types of docetaxel-loaded nanoparticles. For all surfactants, an initial burst was observed. After that, the release of docetaxel occurred at a constant rate. Obviously, docetaxel was released more slowly from the nanoparticles formulated with TPGS and Poloxamer 188 than those formulated with polyvinyl alcohol. Diffusion of the drug, erosion, swelling of the polymer matrix, and degradation of the polymer are the main mechanisms for drug release. Since the degradation of PLGA is slow, ${ }^{18}$ release of docetaxel from the nanoparticles would depend mainly on drug diffusion and matrix erosion. In such cases, the size, hardness, and porosity of the nanoparticles should significantly affect the release properties. Scanning electron microscopy indicated that all types of nanoparticles had a smooth surface, which supported the slow release of drug. ${ }^{21}$ Moreover, size is also an important factor that determines the release rate, and the nanoparticles prepared by TPGS, polyvinyl alcohol, and Poloxamer 188 were similar in size. ${ }^{21}$ TPGS and Poloxamer 188 are bulky and have a large surface area. Thus, the nanoparticles emulsified by TPGS exhibit slower in vitro release than those with polyvinyl alcohol, which has different surface properties. ${ }^{21}$ Figure 7B shows the in vitro release kinetics for the three types of docetaxel-loaded nanoparticles. For all organic

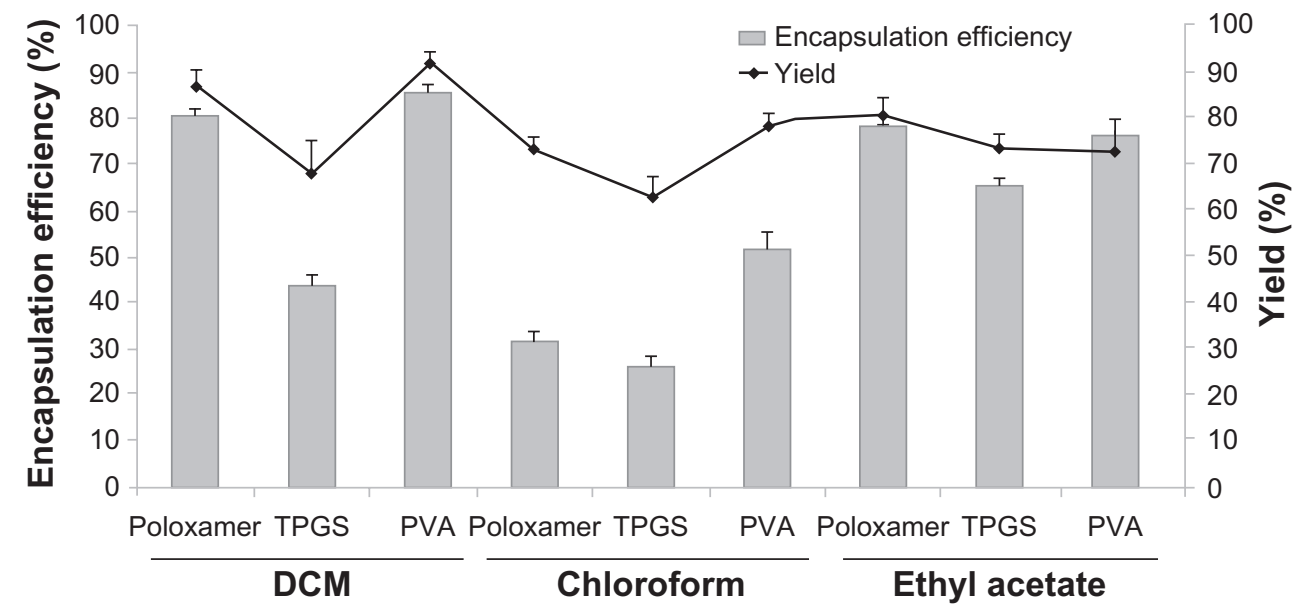

Figure 5 Encapsulation efficiency and yield of each formulation with various organic solvents and surfactants. Abbreviations: PVA, polyvinyl alcohol; TPGS, D- $\alpha$-tocopheryl polyethylene glycol 1000 succinate; DCM, dichloromethane. 
Table 5 X-ray photoelectron spectroscopy for surface chemistry

\begin{tabular}{|c|c|c|c|c|c|c|c|}
\hline \multirow[t]{2}{*}{ Sample } & \multicolumn{4}{|c|}{ XPS CIs envelope ratio (\%) } & \multicolumn{3}{|c|}{ XPS elemental ratio (\%) } \\
\hline & $\mathrm{C}-\mathrm{C}$ & C-O & $\mathrm{C}=\mathrm{O}$ & $\mathrm{O}-\mathrm{C}=\mathrm{O}$ & C & $\mathbf{N}$ & $\mathbf{0}$ \\
\hline RG502 & 1.6 & 13.8 & 23.5 & 18.9 & 57.8 & & 42.2 \\
\hline PVA & 31.4 & 30.8 & & 4.1 & 66.2 & & 33.8 \\
\hline TPGS & 29.4 & 39.2 & & 1.8 & 70.3 & & 29.7 \\
\hline Docetaxel & 49.0 & 16.2 & 3.6 & 4.4 & 73.2 & 2.0 & 25.1 \\
\hline Sucrose & 4.8 & 40.4 & 6.4 & & 51.6 & & 48.4 \\
\hline Physical mixture & & & & & & 0.5 & 43.1 \\
\hline Blank nanoparticles & 28.7 & 18.3 & 5.6 & 11.4 & 64.1 & & 35.9 \\
\hline Docetaxel PLGA nanoparticles & 29.3 & 17.5 & 7.3 & 8.1 & 62.2 & 0.4 & 37.4 \\
\hline
\end{tabular}

Abbreviations: SD, standard deviation; DCM, dichloromethane; PLGA, polylactic-co-glycolic acid; PVA, polyvinyl alcohol; TPGS, D- $\alpha$-tocopheryl polyethylene glycol I000 succinate; XPS, x-ray photoelectron spectroscopy.

solvents, an initial burst was observed. After that, the release of docetaxel occurred at a constant rate. The mean release rate of ethyl acetate was slower than that of dichloromethane and chloroform. However, the three organic solvents did not affect the slow-release kinetics.

\section{Conclusion}

The influence of various processing parameters on particle size and drug encapsulation efficiencies was systematically investigated. It was concluded that formulation variables can be developed in order to enhance the encapsulation of a hydrophobic drug into PLGA nanoparticles using an o/w emulsification method. With the optimal parameters, small particles with a spherical shape could be obtained. The size of the nanoparticles could be adjusted by modifying various process parameters explicitly, such as surfactant type and concentration, sonication parameters, evaporation parameters, and centrifugation parameters. To obtain a smaller particle, $0.2 \%$ polyvinyl alcohol, $0.03 \%$ TPGS, or $2 \%$ Poloxamer 188, five minutes of sonication time, $130 \mathrm{~W}$ sonication power, evaporation with magnetic stirring, and centrifugation at $8000 \mathrm{rpm}$, were selected. The encapsulation efficiency of drug was dependent on different formulation factors, such as mixing order, sonication time and power, drug loading amount, type of surfactant, and organic solvent. To increase the encapsulation efficiency in the nanoparticles, factors were varied from 2-5 minutes of sonication time, 70-130 W sonication power, and a 5-25 mg drug loading amount. As a result, five minutes of sonication time, $130 \mathrm{~W}$ sonication power, and a drug loading amount of $10 \mathrm{mg}$ were selected. Docetaxel showed different release from the nanoparticles over a 28-day period with different types of organic solvents and surfactants. In the release kinetics,

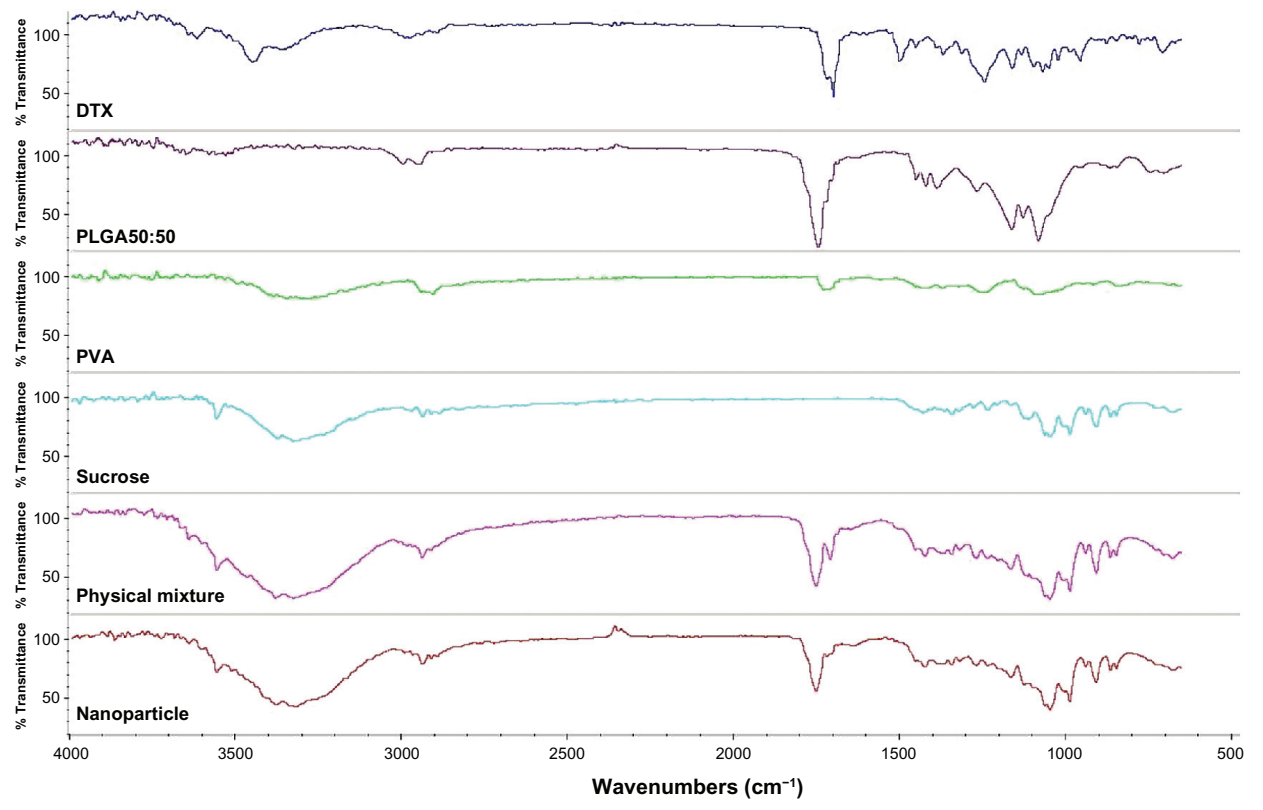

Figure 6 Fourier transform infrared spectroscopy for surface chemistry.

Abbreviations: PVA, polyvinyl alcohol; DTX, docetaxel; PLGA, polylactic acid-co-glycolic acid. 

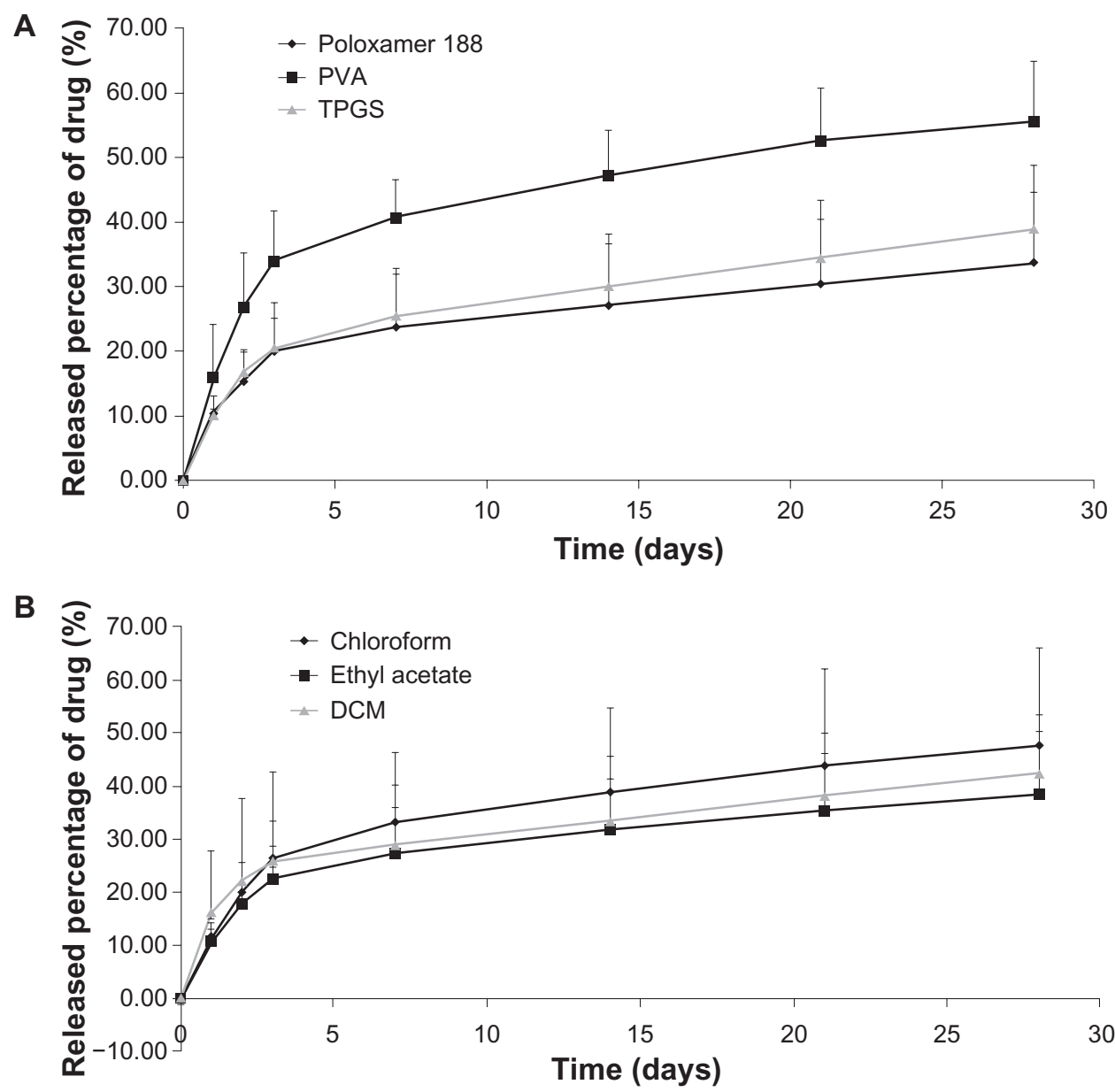

Figure $\mathbf{7}$ In vitro release kinetics with (A) various surfactants and (B) organic solvents.

Abbreviations: PVA, polyvinyl alcohol; TPGS, D- $\alpha$-tocopheryl polyethylene glycol 1000 succinate; DCM, dichloromethane.

$20.83 \%, 40.07 \%$, and $51.5 \%$ of docetaxel were released in 28 days from nanoparticle with Poloxamer 188, TPGS, and polyvinyl alcohol, respectively. Comparing the release kinetics with various surfactants, TPGS and Poloxamer 188 had slower release kinetics than polyvinyl alcohol. X-ray photoelectron spectroscopy demonstrated that residual drug remained on the surface. This study confirmed that variable factors affect mean nanoparticle diameter, encapsulation efficiency, and release kinetics.

\section{Acknowledgments}

This work was supported by the Priority Research Centers Program (2009-0093815) and the Basic Science Research Program (2009-0067380) through the National Research Foundation of Korea funded by the Ministry of Education, Science, and Technology.

\section{Disclosure}

The authors declare no conflicts of interest in this work.

\section{References}

1. Denis JN, Greene AE, Guenard D, et al. Highly efficient, practical approach to natural taxol. J Am Chem Soc. 1988;110:5917-5919.

2. Mangatal L, Adeline MT, Guenard D, et al. Application of the vicinal oxyamination reaction with asymmetric induction to the hemisynthesis of taxol and analogues. Tetrahedron. 1989;45:4177-4190.

3. Gelderblom H, Verweij J, Nooter K, et al. Cremophor EL: the drawbacks and advantages of vehicle selection for drug formulation. Eur J Cancer. 2001;37:1590-1598.

4. Immordino ML, Brusa P, Arpicco S, et al. Preparation, characterization, cytotoxicity and pharmacokinetics of liposomes containing docetaxel. $J$ Control Release. 2003;91:417-429.

5. Alexopoulos A, Karamouzis MV, Stavrinides H, et al. Phase II study of pegylated liposomal doxorubicin (caelyx) and docetaxel as first-line treatment in metastatic breast cancer. Ann Oncol. 2004;15: 891-895.

6. Grosse PY, Bressolle F, Pinguet F. In vitro modulation of doxorubicin and docetaxel antitumoral activity by methyl- $\beta$-cyclodextrin. Eur J Cancer. 1998;34:168-174

7. Mu L, Feng SS. A novel controlled release formulation for the anticancer drug paclitaxel $\left(\right.$ Taxol $\left.^{\circledR}\right)$ : PLGA nanoparticles containing vitamine E TPGS. J Control Release. 2003;86:33-48.

8. Chernysheva YV, Babak VG, Kildeeva NR, et al. Effect of the type of hydrophobic polymers on the size of nanoparticles obtained by emulsification-solvent evaporation. Mendeleev Communications. 2003;13: $65-68$. 
9. Holzer M, Vogel V, Mäntele W, et al. Physico-chemical characterisation of PLGA nanoparticles after freeze-drying and storage. Eur J Pharm Biopharm. 2009;72:428-437.

10. Yan F, Zhang C, Zheng Y, et al. The effect of Poloxamer 188 on nanoparticle morphology, size, cancer cell uptake, and cytotoxicity. Nanomedicine. 2010;1:170-178.

11. Fonseca CT, Simoes SG, Gaspar RG. Paclitaxel-loaded PLGA nanoparticles: preparation, physicochemical characterization and in vitro anti-tumoral activity. $J$ Control Release. 2002;83:273-286.

12. Manchanda R, Fernandez-Fernandez A, Nagesetti A, McGoron AJ. Preparation and characterization of a polymeric (PLGA) nanoparticulate drug delivery system with simultaneous incorporation of chemotherapeutic and thermo-optical agents. Colloids Surf B Biointerfaces. 2010; 75:260-267.

13. Galindo-Rodriguez S, Allemann E, Fessi H, et al. Physicochemical parameters associated with nanoparticle formulation in the salting-out, emulsification-diffusion and nanoprecipitation method. Pharm Res. 2004;21:1428-1439.

14. Mainardes RM, Evangelista RC. PLGA nanoparticles containing praziquantel: effect of formulation variables on size distribution. Int $J$ Pharm. 2005;290:137-144.
15. Kwon HY, Lee JY, Choi SW, et al. Preparation of PLGA nanoparticles containing estrogen by emulsification-diffusion method. Colloids Surf A Physicochem Eng Asp. 2001;182:123-130.

16. Feng SS, Huang GF. Effects of emulsifiers on the controlled release of paclitaxel (Taxol) from nanospheres of biodegradable polymers. $J$ Control Release. 2001;71:53-69.

17. Mu L, Feng SS. Vitamin E TPGS used as emulsifier in the solvent evaporation/extraction technique for fabrication of polymeric nanospheres for controlled release of paclitaxel (Taxol). J Control Release. 2002;80:129-144.

18. Scholes PD, Coombes AG, Illum L, et al. Detection and determination of surface levels of poloxamer and PVA surfactant on and the biodegradable nanospheres using SSIMS and XPS. J Control Release. 1999;59:261-278.

19. Musumeci T, Ventura CA, Giannone I, et al. PLA/PLGA nanoparticles for sustained release of docetaxel. Int J Pharm. 2006;325:172-179.

20. Coates J. Interpretation of infrared spectra, a practical approach. In: Meyers RQ, editor. Encyclopedia of Analytical Chemistry. Chichester, UK: John Wiley \& Sons Ltd; 2000.

21. Astete CE, Sabliov CM. Synthesis and characterization of PLGA nanoparticles. J Biomater Sci Polym Ed. 2006;173:247-289.
International Journal of Nanomedicine

\section{Publish your work in this journal}

The International Journal of Nanomedicine is an international, peerreviewed journal focusing on the application of nanotechnology in diagnostics, therapeutics, and drug delivery systems throughout the biomedical field. This journal is indexed on PubMed Central, MedLine, CAS, SciSearch $\AA$, Current Contents ${ }^{\circledR} /$ Clinical Medicine,

\section{Dovepress}

Journal Citation Reports/Science Edition, EMBase, Scopus and the Elsevier Bibliographic databases. The manuscript management system is completely online and includes a very quick and fair peer-review system, which is all easy to use. Visit http://www.dovepress.com/ testimonials.php to read real quotes from published authors. 